\title{
Computer-assisted measurement of primary tumor area is prognostic of recurrence-free survival in stage IB melanoma patients
}

\author{
Brooke E Rosenbaum ${ }^{1,4}$, Christine N Schafer ${ }^{1,4}$, Sung Won Han ${ }^{2}$, Iman Osman ${ }^{1}$, \\ Hua Zhong ${ }^{3,5}$ and Nooshin Brinster ${ }^{1,5}$ \\ ${ }^{1}$ The Ronald O. Perelman Department of Dermatology, New York University School of Medicine, New York, \\ NY, USA; ${ }^{2}$ Department of Industrial Management Engineering, Korea University, Seoul, Republic of Korea \\ and ${ }^{3}$ Department of Population Health, New York University School of Medicine, New York, NY, USA
}

\begin{abstract}
Current staging guidelines are insufficient to predict which patients with thin primary melanoma are at high risk of recurrence. Computer-assisted image analysis may allow for more practical and objective histopathological analysis of primary tumors than traditional light microscopy. We studied a prospective cohort of stage IB melanoma patients treated at NYU Langone Medical Center from 2002 to 2014. Primary tumor width, manual area, digital area, and conformation were evaluated in a patient subset via computer-assisted image analysis. The associations between histologic variables and survival were evaluated using Cox proportional hazards model. Logistic regressions were used to build a classifier with clinicopathological characteristics to predict recurrence status. Of the 655 patients with stage IB melanoma studied, a subset of 149 patient tumors (63 recurred, 86 did not recur) underwent computer-assisted histopathological analysis. Increasing tumor width (hazard ratios (HR): 1.17, $P=0.01$ ) and digital area (HR: 1.08, $P<0.01$ ) were significantly associated with worse recurrence-free survival, whereas non-contiguous conformation (HR: $0.57, P=0.05$ ) was significantly associated with better recurrence-free survival. The novel histopathological classifier composed of digital area, conformation, and baseline variables effectively distinguished recurrent cases from non-recurrent cases (AUC: $0.733,95 \%$ confidence interval (Cl): 0.647-0.818), compared to the baseline classifier alone (AUC: 0.635 , $95 \% \mathrm{Cl}$ : $0.545-$ 0.724). Primary tumor cross-sectional area, width, and conformation measured via computer-assisted analysis may help identify high-risk patients with stage IB melanoma.

Modern Pathology (2017) 30, 1402-1410; doi:10.1038/modpathol.2017.64; published online 21 July 2017
\end{abstract}

Melanoma incidence continues to increase at a rate of $3-7 \%$ per year. ${ }^{1-3}$ Increased public awareness and screening efforts have led to substantial stage migration at diagnosis- $\sim 70 \%$ of newly diagnosed melanomas are now thin (Breslow thickness $\leq 1 \mathrm{~mm}$ ), invasive lesions. ${ }^{4}$ Although most patients diagnosed with thin primary melanoma have excellent outcomes, it is estimated that $3-5 \%$ of patients with thin melanoma, the majority of whom are classified as stage IB, will go on to develop metastatic

Correspondence: BE Rosenbaum, MD, The Ronald O. Perelman Department of Dermatology, New York University School of Medicine, 550 First Avenue, Smilow Building 401, New York, NY 10016, USA.

E-mail: Brooke.Rosenbaum@med.nyu.edu

${ }^{4}$ These authors contributed equally to this paper.

${ }^{5}$ These authors contributed equally to this paper.

Received 12 November 2016; revised 2 May 2017; accepted 3 May 2017; published online 21 July 2017 disease. $^{5-7}$ According to the 2009 American Joint Committee on Cancer (AJCC) staging system, stage IB melanomas are defined as those thinner than $1 \mathrm{~mm}$ with the presence of either ulceration or at least one mitotic figure per $\mathrm{mm}^{2}$, or as those with tumor thickness of 1.01 to $2 \mathrm{~mm}$, and without ulceration. ${ }^{8,9}$ The 10-year survival rate associated with stage IB melanoma is $\sim 85 \% .{ }^{9}$ Given that a substantial portion of stage IB melanomas will progress, identification of clinical and pathologic variables associated with high-risk stage IB disease is crucial.

The current AJCC staging system is insufficient to predict which patients with stage IB melanoma are at highest risk of recurrence. The AJCC criteria described above allow for substantial histopathological heterogeneity within stage IB melanoma patients. Further stratification of stage IB patients based on histopathological phenotypes may help identify high- and low-risk patient subsets. Previous studies have demonstrated significant associations of 
increasing tumor volume with higher risk of sentinel lymph node metastases and worse survival outcomes in melanoma. ${ }^{10-13}$ Given the logistical constraints of measuring tumor volume, most of these studies estimated volume based on the mathematical approximation for a hemiellipsoid, which typically require serial measurements from multiple thin tumor sections. ${ }^{10,12}$ These methods can be difficult to standardize and exceedingly time-consuming, thus limiting their practicality in the clinical setting. ${ }^{10-16}$ Recent advances in digital pathology software, however, may now allow more rigorous and efficient histopathological tumor analysis, providing an opportunity to re-evaluate multidimensional tumor measurements in thin primary melanomas. ${ }^{17-19}$

To improve risk stratification of thin melanoma, we investigated the prognostic value of multidimensional primary tumor measurements (eg, crosssectional area and width) in a large, prospective cohort of stage IB melanoma patients. We postulated that multidimensional primary tumor measurements may be useful in predicting which patients with thin primary melanoma are at highest risk of recurrence. In addition, the use of digital pathology software tools may allow for more practical and objective evaluation of multidimensional primary tumor measurements than those methods utilized in prior studies.

\section{Materials and methods}

\section{Study Population}

We studied a cohort of patients who presented to the NYU Langone Medical Center with AJCC stage IB melanoma from August 2002 to May 2014 and were prospectively enrolled in the Interdisciplinary Melanoma Cooperative Group, a prospective clinicopathological biospecimen database. ${ }^{20}$ This study was approved by the NYU Institutional Research Board (Study \#10362) and all patients provided informed, written consent at the time of enrollment. For patients referred from outside institutions, the diagnosis of primary melanoma was confirmed by the analysis of biopsy specimens by two NYU pathologists. All primary melanoma patients underwent surgical treatment of the initial primary tumor.

Patient demographics, clinical data, and primary tumor characteristics were obtained from clinical charts and pathology reports. Variables assessed included patient sex, age at stage IB diagnosis, primary tumor thickness, primary tumor ulceration, mitotic rate (per $\mathrm{mm}^{2}$ ), and histological subtype. Date of stage IB diagnosis was defined as the biopsy date of the primary tumor. Recurrence-free survival was defined as the time from the date of pathological diagnosis of stage IB melanoma to the first recorded date of regional or distant metastases. Overall survival (OS) was defined as the time from the date of pathological diagnosis of stage IB melanoma until death. Recurrence and survival information were collected by active, prospective follow-up of all patients enrolled in the database.

\section{Computer-Assisted Histopathological Analysis}

A subset of patients was selected for further examination of additional histological variables via computer-assisted image analysis. This group consisted of all patients with stage IB melanoma whose disease later recurred, along with a subset of patients with non-recurrent disease, matched for age, gender, histopathological subtype, thickness, ulceration, and mitotic rate. Patients were excluded if the corresponding H\&E-stained slide of their primary tumor was unavailable. Primary tumor slides were scanned into digital images and independently reviewed by the Interdisciplinary Melanoma Cooperative Group dermatopathologist (NB). Slides were examined using computer-assisted analysis (Aperio, Vista, CA, USA) to confirm primary tumor characteristics, as well as assess tumor width, manual tumor area, digital tumor area, and tumor conformation. Tumors were considered 'contiguous', if the majority of the tumor was present in one coalesced mass, whereas those divided into multiple, separate aggregates were considered 'non-contiguous.' Tumor width (mm) was measured in the invasive dermal component in the greatest horizontal dimension. In non-contiguous tumors, the sum of widths of individual dermal tumors was measured. Manual tumor area $\left(\mathrm{mm}^{2}\right)$ was estimated by multiplying the Breslow thickness (mm) by the width ( $\mathrm{mm}$ ) of the invasive dermal component. To calculate digital tumor area $\left(\mathrm{mm}^{2}\right)$, the invasive dermal component of the tumors were manually outlined by the dermatopathologist and the tumor area calculated by the software.

\section{Statistical Analysis}

We tested the association between histologic variables and recurrence-free survival, and OS using Cox univariate and multivariate analysis; only variables significant in univariate analysis were included in the multivariate model. Linear regression analysis was performed to assess the relationship between manual and digital area for all patients, including patients subdivided by conformation. Using the research cohort, we first examined the differences in clinicopathological characteristics between patients with recurrent melanoma and those with non-recurrent disease using Wilcoxon/Mann-Whitney nonparametric test for continuous variables and Fisher's exact test for categorical variables. We then use logistic regressions to build classifier with clinicopathological characteristics to predict recurrence status. The candidate predictors of the baseline model were restricted to age, gender, histological subtype, thickness, mitoses (yes or no), and 
ulceration status. The candidate predictors of the novel histopathological model include the selected variables in the baseline model and the candidate from the novel histopathological predictors, including width, conformation status (contiguous or noncontiguous), and digital area or manual area. Both models were selected by minimizing Akaike's information criterion of the logistic regression. We used a 10-fold cross-validation approach to obtain an unbiased estimate of the area under the receiver operating characteristic (ROC) curve (AUC) of the selected model and its 95\% confidence interval (CI). The linear combination of the selected model predictors weighted by regression coefficients was defined as the risk score. A risk score cutoff using the Youden Index of the ROC curve was chosen to separate patients into high- and low-recurrence risk groups. Kaplan-Meier survival curves and log-rank tests were used to compare the recurrence-free survival of the two groups. All statistical tests were performed by in $\mathrm{R}$ including packages 'survival', 'ROCR', 'cvAUC', and 'OptimalCutpoints'.

\section{Results}

\section{Patient Characteristics}

Table 1 displays the demographic and primary tumor characteristics of the patient cohort $(n=655)$. Median follow-up was 4.4 years. The median age at stage IB diagnosis was 58 years and $57.1 \%$ of patients were male. The median primary tumor thickness and mitotic rate were $0.98 \mathrm{~mm}$ and 1 per $\mathrm{mm}^{2}$, respectively. Ulceration was present in $4.3 \%$ of primary tumors. The most common histological subtype was superficial spreading (67.3\%). Overall, $77(11.8 \%)$ patients experienced a recurrence of their disease, whereas $578(88.2 \%)$ of patients remained in remission throughout the study period.

\section{Increasing Thickness, Mitotic Rate, and Ulceration are} Significantly Associated with Survival Outcomes

We performed survival analysis to assess the relationship between patient baseline variables and survival outcomes. In univariate analysis, increasing Breslow thickness (hazard ratios (HR): 4.63, $P<0.01$ ), presence of ulceration (HR: 2.27, $P=0.05$ ), and increasing mitotic rate (HR: 1.14, $P<0.01)$ were significantly associated with worse Interdisciplinary Melanoma Cooperative Group Interdisciplinary Melanoma Cooperative Group recurrence-free survival. Increasing age (HR: 1.02, $P=0.04$ ), Breslow thickness (HR: 3.26, $P<0.01$ ), and mitotic rate (HR: 1.14, $P<0.01$ ) were significantly associated with worse OS. In multivariate analysis, Breslow thickness (HR: 4.13, $P<0.01$ ) and ulceration (HR: 2.44, $P=0.04$ ) remained significantly associated with worse recurrence-free survival. Breslow thickness (HR: 2.54, $P<0.01$ ) and

Table 1 Baseline patient and tumor characteristics $(n=655)$

Characteristic

Sex, n $(\%)$

Male

Female

Age (years), median (s.d.)

Thickness (mm), median (s.d.)

Ulceration, n (\%)

Present

Absent

Mitotic rate (per $\mathrm{mm}^{2}$ ), median (s.d.)

Mitoses, n (\%)

No

Yes

Histological subtype, n (\%)

Superficial spreading

Nodular

Other

Recurrence, n (\%)

Not recurred

Recurred

$77(11.8)$

Type of recurrence, n (\%)

Local

Only local

Regional

Only regional

Distant

Only distant

Distant+local

Distant+regional

Distant+regional+local

Unknown

Death, n $(\%)$

Alive

Dead

$578(88.4)$

$76(11.6)$

Abbreviation: s.d., standard deviation.

Local = tumor recurrence within $2 \mathrm{~cm}$ of the surgical scar following definitive excision of a primary melanoma with sufficient surgical margins.

Regional = tumor recurrence in the regional lymph node basin or that is intralymphatic (satellites or in transit metastases).

Distant $=$ tumor recurrence in distant lymph nodes, skin or subcutaneous tissue $>2 \mathrm{~cm}$ from the original primary tumor site, or distant organs.

increasing mitotic rate (HR: $1.10, P=0.02)$ remained significantly associated with worse OS.

\section{Primary Tumor Width, Digital Area, and Conformation are Prognostic of Worse Recurrence-Free Survival}

A subset of 149 patients were examined for digital histopathological variables and associations with survival (Table 2). Fourteen of the 77 patients with recurrent disease were excluded from subanalysis due to unavailability of primary tumor slides for review. In this group, $63(42.3 \%)$ patients had disease that later recurred with regional or distant metastases, whereas $86(57.7 \%)$ patients had disease 
Table 2 Baseline patient and primary tumor characteristics of the patients that underwent digital histopathological analysis ( $n=149$ ), only those whose disease recurred $(n=63)$, and only those with non-recurrent disease $(n=86)$

\begin{tabular}{|c|c|c|c|c|}
\hline Characteristic & Total $(\mathrm{n}=149)$ & Recurred (n=63) & Did not recur $(\mathrm{n}=86)$ & $\mathrm{P}$-value \\
\hline \multicolumn{5}{|l|}{ Sex, n $(\%)$} \\
\hline Male & $94(63.1)$ & $39(61.9)$ & $55(64)$ & \multirow[t]{2}{*}{0.93} \\
\hline Female & $55(36.9)$ & $24(38.1)$ & $31(36)$ & \\
\hline Age (years), median (s.d.) & $60(15.8)$ & $58(15.8)$ & $62(15.7)$ & 0.16 \\
\hline Thickness (mm), median (s.d.) & $1.14(0.43)$ & $1.10(0.48)$ & $1.15(0.39)$ & 0.88 \\
\hline \multicolumn{5}{|l|}{ Ulceration, $\mathrm{n}(\%)$} \\
\hline Present & $14(9.6)$ & $6(9.8)$ & $8(9.4)$ & \multirow[t]{2}{*}{1} \\
\hline Absent & $132(90.4)$ & $55(90.2)$ & $77(90.6)$ & \\
\hline \multicolumn{5}{|l|}{ Mitoses, n (\%) } \\
\hline No & $5(5.8)$ & $16(25.4)$ & $21(14.1)$ & \multirow[t]{2}{*}{$<0.01$} \\
\hline Yes & $81(94.2)$ & $47(74.6)$ & $128(85.9)$ & \\
\hline Mitotic rate (per $\mathrm{mm}^{2}$ ), median (s.d.) & $2(2.7)$ & $3(2.4)$ & $2(2.9)$ & 0.73 \\
\hline \multicolumn{5}{|l|}{ Histological subtype, n (\%) } \\
\hline Superficial spreading & $89(61)$ & 37 (59.7) & $52(61.9)$ & \multirow[t]{3}{*}{0.50} \\
\hline Nodular & $47(32.2)$ & $19(30.6)$ & $28(33.3)$ & \\
\hline Other & $10(6.8)$ & $6(9.7)$ & $4(4.8)$ & \\
\hline \multicolumn{5}{|l|}{ Novel histopathological variables } \\
\hline Width (mm), median (s.d.) & $3.73(1.88)$ & $4.19(2.01)$ & $3.41(1.73)$ & 0.03 \\
\hline Manual area $\left(\mathrm{mm}^{2}\right)$, median (s.d.) & $3.97(3.32)$ & $4.38(3.64)$ & $3.70(3.03)$ & 0.14 \\
\hline Digital area $\left(\mathrm{mm}^{2}\right)$, median (s.d.) & $2.10(2.97)$ & $2.47(4)$ & $1.90(1.69)$ & 0.01 \\
\hline \multicolumn{5}{|l|}{ Conformation, $n(\%)$} \\
\hline Contiguous & $95(63.8)$ & $45(71.4)$ & $50(58.1)$ & \multirow{2}{*}{0.14} \\
\hline Non-contiguous & $54(36.2)$ & $18(28.6)$ & $36(41.9)$ & \\
\hline
\end{tabular}

Abbreviation: s.d., standard deviation.

did not recur. There were no significant differences in sex, age at diagnosis, tumor thickness, ulceration, or mitotic rate between patients with recurrent melanoma and those with non-recurrent disease.

The digital histopathological variables evaluated are illustrated in Figure 1. Manual area was estimated by multiplying the Breslow thickness by the width of the tumor (Figure 1a), whereas digital area was calculated via the Aperio software algorithm (Figure 1b). Tumors were classified as contiguous or non-contiguous, as displayed in Figure $1 \mathrm{~b}$ and c. Tumor width was measured as the cummulative sum of widths of the tumor's invasive dermal components (Figure 1d), respectively. The median width, digital area, and manual area measurements of the entire research cohort were $3.73 \mathrm{~mm}, 2.10 \mathrm{~mm}^{2}$, and $3.97 \mathrm{~mm}^{2}$, respectively (Table 2). With respect to tumor conformation, $63.8 \%$ of primary tumors were considered contiguous, whereas $36.2 \%$ were classified as noncontiguous. Primary tumors that later recurred were significantly larger in width $(4.41 \mathrm{~mm}$ vs $4.19 \mathrm{~mm}$; $P=0.03)$ and digital area $\left(2.47 \mathrm{~mm}^{2}\right.$ vs $1.90 \mathrm{~mm}^{2}$; $P=0.01$ ) than those that did not recur. However, no significant differences in manual area $(P=0.14)$ or tumor conformation $(P=0.14)$ was observed between recurrent and non-recurrent groups.

To assess the relationship between digital histopathological variables and survival, statistical analysis was performed by Cox proportional hazard ratios (Table 3). Multivariate analysis demonstrated that increasing tumor width (HR: 1.20, $P<0.01$ ) and digital area (HR: 1.08, $P<0.01$ ) were significantly associated with worse recurrence-free survival, whereas non-contiguous conformation (HR: 0.55, $P=0.05$ ) was significantly associated with better recurrence-free survival. There was a trend towards worse recurrence-free survival with increasing manual area, but this association did not reach significance (HR: 1.08, $P=0.07$ ). No digital histopathological variables demonstrated a significant association with OS.

\section{Novel Histopathological Signature Predicts Recurrence}

Among the 149 patients in the research cohort, three were removed due to missing ulceration status, and one was removed due to missing digital area measurement. Using the baseline variables, we first developed a baseline prognostic model of melanoma recurrence, using the parameters of age at stage $1 \mathrm{~b}$ diagnosis, thickness, and mitoses (yes or no). We then built the prognostic model with the addition of novel histopathological predictors. This model includes digital area and conformation status (contiguous or non-contiguous) in addition to the variables in the baseline model. The novel histopathological classifier displayed an area under the ROC curve (AUC) of 0.733 (95\% CI: 0.647-0.818) estimated via the 10 -fold cross-validation approach, 

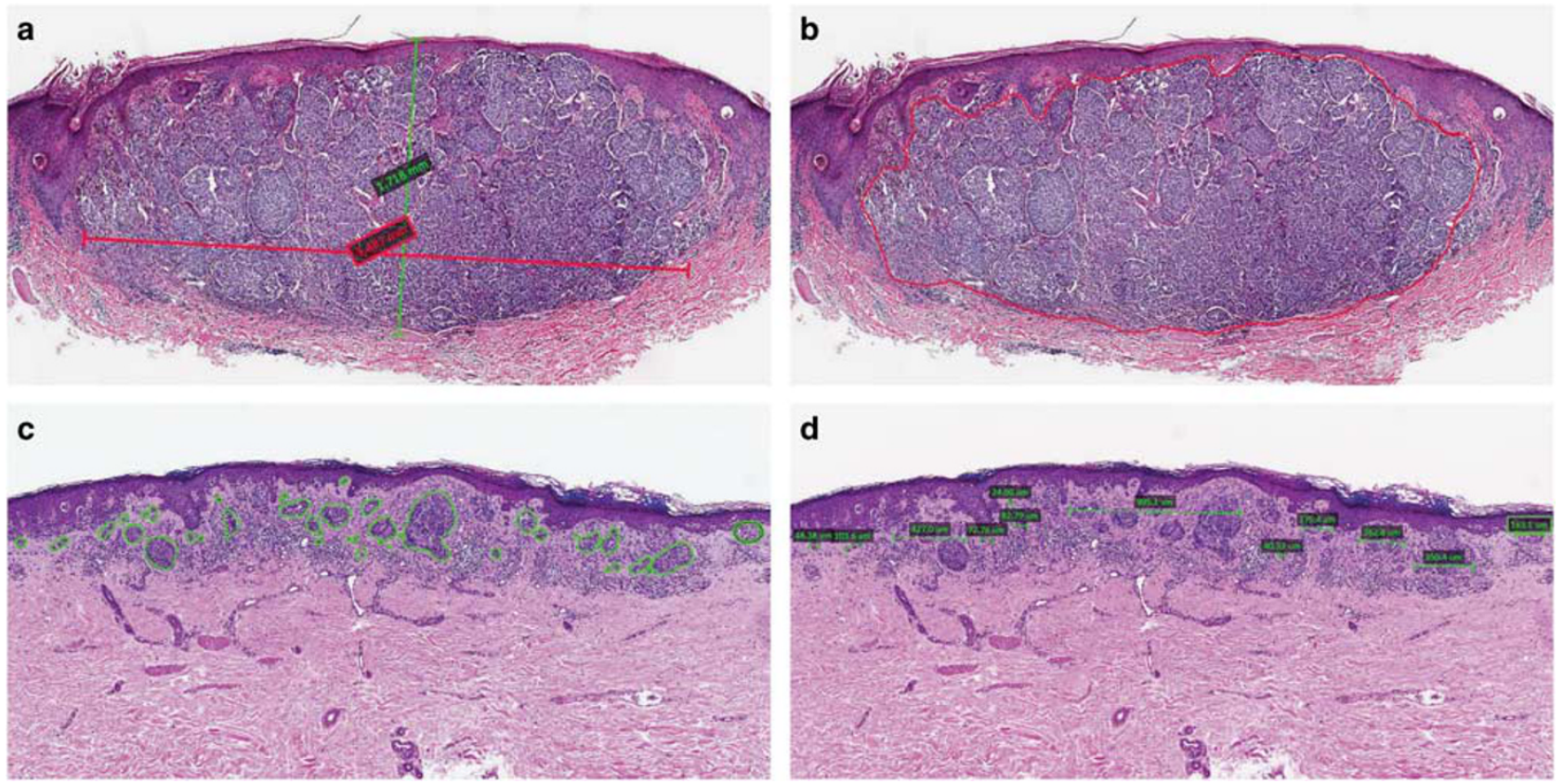

Figure 1 Primary tumor H\&Es demonstrating digital histopathological variables. (a) Manual area was estimated by multiplying the Breslow thickness by the width of the invasive dermal tumor (thickness $1.72 \mathrm{~mm}$, width $3.49 \mathrm{~mm}$, manual area $5.99 \mathrm{~mm}^{2}$ ). To measure digital area, the invasive dermal tumor was manually outlined and calculated by the Aperio software algorithm, as demonstrated in a (b) contiguous (digital area $4.99 \mathrm{~mm}^{2}$ ) and a (c) non-contiguous (digital area $0.36 \mathrm{~mm}^{2}$ ). (d) Tumor width was measured as the cumulative sum of widths of the tumor's invasive dermal components in the greatest horizontal dimension (width $2.75 \mathrm{~mm}$ ).

Table 3 Univariate and multivariate analyses of primary tumor width, manual area, digital area, and conformation with recurrence-free and OS $(n=149)$

\begin{tabular}{|c|c|c|c|c|}
\hline & \multicolumn{2}{|c|}{ Univariate analysis } & \multicolumn{2}{|c|}{ Multivariate analysis ${ }^{\mathrm{a}}$} \\
\hline & HR $(95 \%$ CI $)$ & P-value & $H R(95 \% C I)$ & $\mathrm{P}$-value \\
\hline \multicolumn{5}{|l|}{ Recurrence-free survival } \\
\hline Width (mm) & $1.17(1.04-1.32)$ & 0.008 & $1.20(1.06-1.37)$ & 0.004 \\
\hline Manual area $\left(\mathrm{mm}^{2}\right)$ & $1.06(1-1.13)$ & 0.069 & $1.08(0.99-1.18)$ & 0.069 \\
\hline Digital area $\left(\mathrm{mm}^{2}\right)$ & $1.08(1.03-1.14)$ & 0.003 & $1.10(1.04-1.17)$ & 0.002 \\
\hline Conformation ${ }^{\mathrm{b}}$ & $0.57(0.32-0.99)$ & 0.046 & $0.55(0.30-1)$ & 0.048 \\
\hline \multicolumn{5}{|l|}{ Overall survival } \\
\hline Width (mm) & $1.05(0.91-1.21)$ & 0.492 & $1.01(0.87-1.17)$ & 0.924 \\
\hline Manual area $\left(\mathrm{mm}^{2}\right)$ & $1.02(0.94-1.10)$ & 0.690 & $0.98(0.89-1.08)$ & 0.641 \\
\hline Digital area $\left(\mathrm{mm}^{2}\right)$ & $1.04(0.97-1.11)$ & 0.305 & $1.02(0.93-1.13)$ & 0.643 \\
\hline Conformation ${ }^{\mathrm{b}}$ & $1.11(0.60-2.06)$ & 0.745 & $1.10(0.57-2.10)$ & 0.782 \\
\hline
\end{tabular}

Abbreviations: CI, confidence interval; HR, hazard ratio.

${ }^{\mathrm{a}}$ Cox model adjusted for gender, age at diagnosis, thickness (mm), mitoses (yes/no) in addition to the variable of interest.

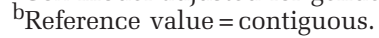

effectively distinguishing recurrent cases from nonrecurrent cases. In contrast, the baseline classifier alone achieved AUCs of 0.635 (0.545, 0.724).

We defined risk scores for individual patients as the linear combination of model predictors weighted by regression coefficients. On the basis of the cutoff value selected by the Youden Index, the sensitivity was $78.7 \%$ and the specificity $72.6 \%$ (Figure 2). For patients predicted to be negative for recurrence by the signature (negative predictive value), 17.6\% $(13 / 74)$ recurred and $82.4 \%(61 / 74)$ patients have not recurred, which supports the clinical sensitivity of the signature. For patients predicted to be positive for recurrence by the signature (positive predictive value), $67.6 \%$ (48/71) patients have recurred and $32.4 \%(23 / 71)$ patients have recurred.

To assess the ability of the signature to stratify patients by recurrence-free survival, high- and lowrisk groups were plotted in Kaplan-Meier survival curves and statistical analysis performed by log-rank tests (Figure 3). High- and low-risk groups displayed clear differences in progression-free survival $(P<0.001)$, but not significant for OR $(P=0.09)$. These analyses underscore the robustness of our classifier in predicting melanoma patient recurrence outcome. 


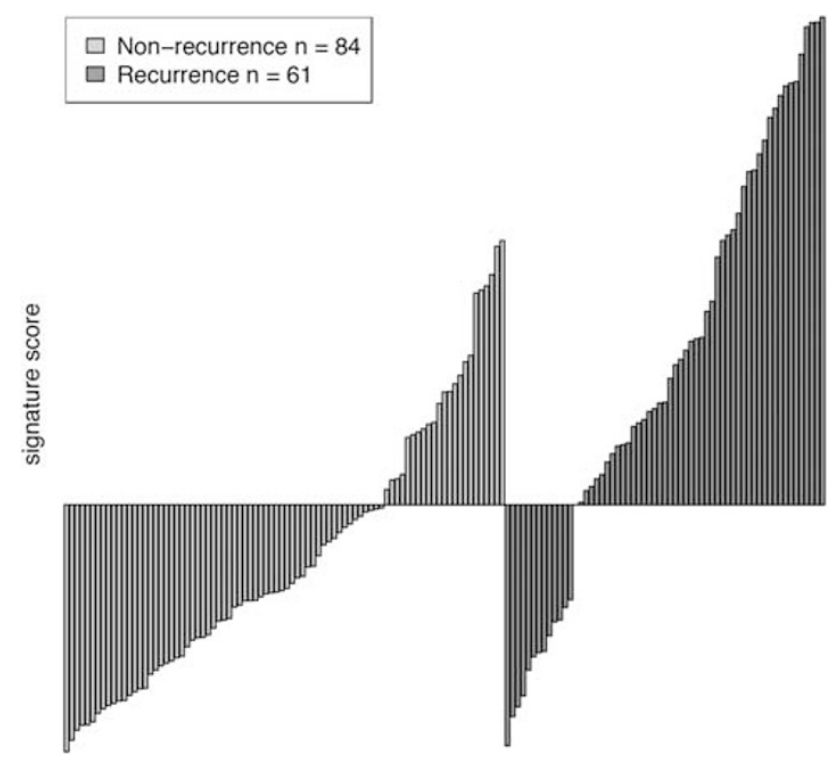

Figure 2 Sensitivity and specificity for prediction of recurrence using a risk score derived from novel histopathological predictive model.

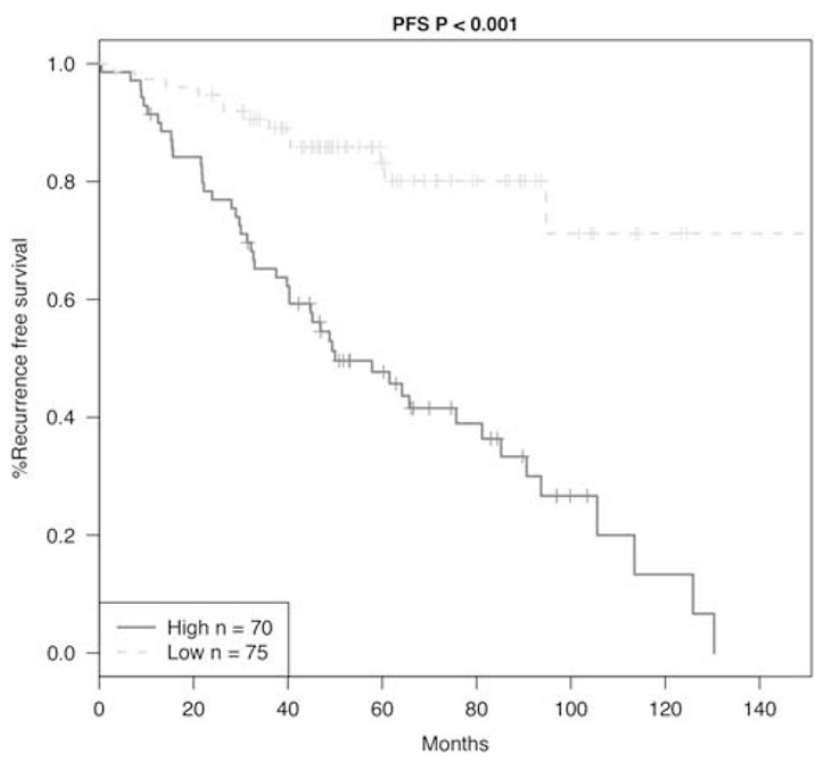

Figure 3 Progression-free survival (PFS) of patients stratified by the novel histopathological predictive model. Using the risk score previously defined by the novel histopathological predictive model, high and low-risk groups were plotted in a Kaplan-Meier survival curve and statistical analysis performed by the logrank test.

We also built a prognostic model including manual area (substituted for digital area) and conformation status (contiguous or non-contiguous) in addition to the variables in the baseline model. This novel histopathological displayed an AUC of 0.691 (95\% CI: $0.602-0.780$ ) estimated via the 10 -fold crossvalidation approach. Therefore, we believe that the signature incorporating digital area is more effective.

\section{Manual and Digital Primary Tumor Area are Highly Correlated, Especially in Contiguous Tumors}

We then investigated whether manual area may be an adequate surrogate for digital area in settings without access to digital pathology software. We evaluated the relationship between manual and digital area, which showed a significant correlation between the two measurements $(P<0.01$; Figure 4a). We then substratified patients into groups of either contiguous $(n=95)$ or non-contiguous $(n=54)$ tumors. Patients with contiguous tumors had a stronger correlation between manual and digital area measurements than did those with non-contiguous tumors (estimate 0.75 vs 0.49 , respectively; $P<0.01$; Figure $4 \mathrm{~b}$ ).

\section{Discussion}

Our study demonstrated the ability of computerassisted histopathological analysis to identify novel prognostic variables in patients with stage IB melanoma. Using computer-assisted image analysis, we identified primary tumor intradermal width, digital area, and conformation as prognostic variables that may help predict which patients with stage IB melanoma are at highest risk of recurrence. Furthermore, our data demonstrated that a signature composed of digital tumor area and tumor conformation, combined with baseline variables, had superior prognostic capabilities compared to baseline variables alone and accurately stratified patients by recurrence-free survival. We also showed that manual tumor area (Breslow thickness $\times$ width), which can be measured digitally or via light microscope, may potentially be a suitable substitute for digital area in clinical settings without access to digital pathology software.

Routine surveillance for patients with thin primary melanoma is cost-ineffective due to their generally low-recurrence rate. ${ }^{21}$ Despite their favorable prognosis, thin melanomas comprise up to $70 \%$ of new melanoma diagnoses and account for over $25 \%$ of all melanoma deaths. ${ }^{4}$ Thus, targeted surveillance for high-risk thin primary melanoma patients has the potential to make a substantial impact on patient mortality. Defining prognostic variables that risk-stratify patients with thin primary melanoma, however, is difficult as these tumors tend to have similar and generally favorable histopathological primary tumor characteristics. Therefore, we utilized computer-assisted image analysis to create a predictive signature of baseline histopathological variables, cross-sectional tumor area, and conformation that effectively risk-stratifies stage IB melanoma patients by recurrence-free survival.

Our results highlight the prognostic utility of tumor cross-sectional area in patients with thin primary melanoma. Previous studies have also demonstrated the prognostic value of digitally calculated tumor area. Two studies of cross-sectional area in primary 

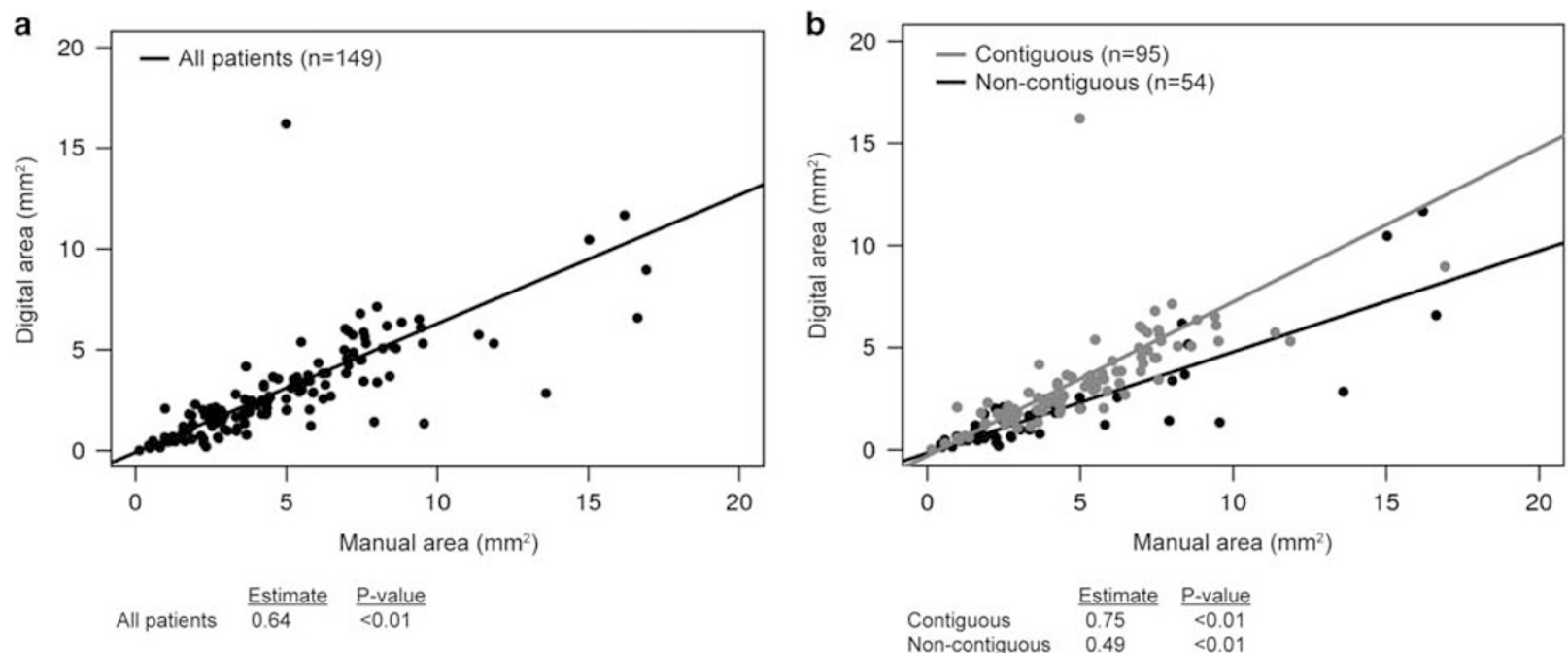

Figure 4 Regression analysis of manual versus digital tumor area for (a) all patients (estimate $0.64, P<0.01$ ) and (b) stratified into those with contiguous tumors and those with non-contiguous tumors (estimate 0.75 and 0.49 , respectively; $P<0.01$ ).

melanomas of all thicknesses (T1-T4) found that increasing tumor area was significantly associated with worse recurrence-free survival and OS. ${ }^{15,16}$ However, in both studies, the prognostic value of cross-sectional area was highly correlated with thickness, and provided little additional prognostic information to the current paradigm. To mitigate the confounding effects of thickness encountered in prior studies, we restricted our study population to patients with stage IB melanoma, limiting the clinical and histopathological heterogeneity of our patient sample. As there is still an inherent range of thicknesses (0$2 \mathrm{~mm}$ ) in stage IB tumors, we then selected a research cohort composed of recurrent and non-recurrent cases matched for Breslow thickness (as well as age, gender, mitoses, and ulceration). Therefore, the association of increasing tumor area with worse recurrence-free survival reported in this study is less likely to be due to confounding with Breslow thickness.

In clinical settings lacking digital pathology software, manual area may be a suitable surrogate for digital area, particularly in patients with contiguous tumors. To extend the generalizability of our results, we also measured the manual area (Breslow thickness $\times$ intradermal width) of each tumor, which can be performed using traditional light microscopy. However, the associations between manual area and recurrence outcomes did not reach statistical significance. Similarly, the predictive model incorporating manual area did not demonstrate superior prognostic capability over that of the baseline model. This may be because manual area is directly dependent on thickness and, thus, was affected by our efforts to control for thickness. Therefore, we recognized that manual area might still provide useful prognostic information when digital pathology software is not available. Linear regression analysis of manual and digital area found a significant correlation between the two measurements in all patients. Further, when patients were substratified by their primary tumor configuration, patients with contiguous tumors had an even stronger correlation between manual and digital tumor area.

Our novel analysis of tumor conformation demonstrated that patients with contiguous tumors had a worse recurrence-free survival than those with noncontiguous tumors. In addition, tumor conformation was included in the novel histopathological signature that accurately stratified patients by recurrencefree survival, and was superior to that of the baseline model. This is the first study to report primary tumor conformation as a potential prognostic variable for melanoma patients. Our findings suggest that there may be underlying biological differences between contiguous and non-contiguous primary tumors. It is also likely, however, that primary tumor conformation is colinear with tumor area and cell proliferation markers, and thus may not offer additional prognostic information to the current staging paradigm. Further investigation of tumor conformation in multivariate models is needed to fully elucidate its prognostic value in thin primary melanoma.

Our study also found the histologic measurement of the primary tumor's intradermal width to be prognostic of recurrence and worse recurrence-free survival. Tumor surface-width (eg, tumor diameter) measured in vivo is typically used as a screening tool in melanoma, where suspicious lesions with a diameter greater than $6 \mathrm{~mm}$ have a higher likelihood of being melanoma than those $<6 \mathrm{~mm} .{ }^{22-24}$ In regard to prognostic value, past studies have noted a correlation between the primary tumor diameter and survival. ${ }^{25-30}$ Breslow's landmark 1970 study found that no primary melanomas $<5 \mathrm{~mm}$ in clinical diameter recurred or metastasized. ${ }^{30}$ In our study, the median width of the invasive dermal component 
determined to be statistically significant in patients with recurrent disease was $4.19 \mathrm{~mm}$, as compared to $3.41 \mathrm{~mm}$ in those who did not recur $(P=0.03)$. We recognize that the width of the invasive component of melanoma is not an exact correlate of clinical diameter. Although the clinical diameter can be accurately measured, the histologic determination of dermal width is potentially confounded by tissue shrinkage, grossing technique (ie, the axis upon which the specimen was sectioned), and variability in the histologic planes of sections examined. ${ }^{31,32}$

Survival analysis of the baseline variables yielded expected results that are consistent with those of the current AJCC guidelines. Interestingly, our analysis of mitotic rate as a continuous variable showed that increasing mitotic rate was independently associated with worse survival. This finding is consistent with multiple recent studies that also demonstrate the powerful independent effect of mitotic rate on patient outcomes, including a multi-institutional study by Thompson et al of 13296 patients with stage I and II melanoma. ${ }^{19,33-36}$ Future staging guidelines should reconsider the use of mitoses as a binary criterion (present vs absent), as there is sufficient evidence that higher mitotic rates are associated with worse outcomes.

There were several limitations to this study. First, we focused our investigation of thin melanoma on patients with AJCC stage IB disease because this patient population is at higher risk of poor outcomes and still lacks clear management guidelines. However, this limited our sample to patients who were histopathologically node-negative or who did not undergo sentinel lymph node biopsy at all, introducing possible sampling bias. This also prevented evaluation of sentinel lymph node positivity as an outcome measure. Second, we evaluated novel histopathological characteristics in a non-random subset of similar recurrent and non-recurrent patients to control for certain variables and increase the relative number of recurrence events. This resulted in a research cohort that was older in age, had thicker primary tumors with higher mitotic rates; however, this is expected due to the increased prevalence of recurrence events. Third, the research cohort was histopathologically reviewed by only one dermatopathologist, thus inter-observer reliability could not be assessed. Finally, we did not split the data into discovery and test cohorts, as there were only 63 stage IB patients whose disease recurred and whose slides were available for review. We attempted to mitigate this limitation, however, by using cross-validation to obtain and unbiased AUC estimate. Therefore, independent validation of our model in a large prospective cohort of stage IB patients is necessary to assess the robustness of our prognostic signature prior to translation into the clinical setting.

Our study highlights the value of computer-assisted histopathological analysis to identify additional prognostic characteristics for patients with thin melanoma. We found primary tumor width, digital area, and conformation to be significantly associated with survival outcomes in stage IB patients, independent of Breslow thickness. Using these data, we developed a novel prognostic histopathological classifier that, in combination with baseline variables, effectively stratifies patients with stage IB melanoma by their risk of recurrence. With this prognostic model, $48.9 \%$ (71/145 in the studied cohort) stage IB patients are predicted at higher risk of recurrence, among whom $67.6 \%(48 / 71)$ patients are correctly predicted and $32.4 \%$ (23/71) patients are falsely classified for the observed follow-up time. The described signature represents a first step towards targeted surveillance-and therefore earlier intervention of recurrence-for high-risk thin primary melanoma patients. We believe such predictive models have the potential to improve survival outcomes for this growing subset of patients with thin melanoma.

\section{Acknowledgments}

This work was supported by the NYU Cancer Institute NCI Cancer Center Support Grant (P30 CA016087) and the Marc Jacobs Campaign to support melanoma research.

\section{Disclosure/conflict of interest}

The authors declare no conflict of interest.

\section{References}

1 Diepgen TL, Mahler V. The epidemiology of skin cancer. Br J Dermatol 2002;146(Suppl 61):1-6.

2 Jemal A, Clegg LX, Ward E, et al. Annual report to the nation on the status of cancer, 1975-2001, with a special feature regarding survival. Cancer 2004;101:3-27.

3 Whiteman DC, Green AC, Olsen CM. The growing burden of invasive melanoma: projections of incidence rates and numbers of new cases in six susceptible populations through 2031. J Invest Dermatol 2016;136: 1161-1171.

4 Linos E, Swetter SM, Cockburn MG, et al. Increasing burden of melanoma in the United States. J Invest Dermatol 2009;129:1666-1674.

5 Kalady MF, White RR, Johnson JL, et al. Thin melanomas: predictive lethal characteristics from a 30-year clinical experience. Ann Surg 2003;238:528-535.

6 Mansson-Brahme E, Carstensen J, Erhardt K, et al. Prognostic factors in thin cutaneous malignant melanoma. Cancer 1994;73:2324-2332.

7 Venna SS, Thummala S, Nosrati M, et al. Analysis of sentinel lymph node positivity in patients with thin primary melanoma. J Am Acad Dermatol 2013;68: 560-567.

8 Balch CM, Soong SJ, Gershenwald JE, et al. Prognostic factors analysis of 17,600 melanoma patients: validation of the American Joint Committee on Cancer melanoma staging system. J Clin Oncol 2001;19: 3622-3634. 
9 Balch CM, Gershenwald JE, Soong SJ, et al. Final version of 2009 AJCC melanoma staging and classification. J Clin Oncol 2009;27:6199-6206.

10 Friedman RJ, Rigel DS, Kopf AW, et al. Volume of malignant melanoma is superior to thickness as a prognostic indicator. Preliminary observation. Dermatol Clin 1991;9:643-648.

11 Panasiti V, Curzio M, Roberti V, et al. Metastatic volume: an old oncologic concept and a new prognostic factor for stage IV melanoma patients. Dermatology 2013;227:55-61.

12 Voss B, Wilop S, Jonas S, et al. Tumor volume as a prognostic factor in resectable malignant melanoma. Dermatology 2014;228:66-70.

13 Walton RG, Kim J, Velasco C, et al. Tumor volume: an adjunct prognostic factor in cutaneous melanoma. Cutis 2014;94:226-230.

14 Gebhart W, Knobler R. Computer-assisted volumetric analysis of cutaneous malignant melanomas. Am J Dermatopathol 1984;6 Suppl:93-95.

15 Temple CF, Huchcroft SA, Hurlbut DJ, et al. Histologic staging in malignant melanoma: cross-sectional area revisited. J Surg Oncol 1998;69:83-87.

16 Smolle J, Okcu A, Hofmann-Wellenhof R, et al. Automated measurement of melanoma cross-sectional area. Am J Dermatopathol 1998;20:155-159.

17 Hipp J, Cheng J, Daignault S, et al. Automated area calculation of histopathologic features using SIVQ. Anal Cell Pathol 2011;34:265-275.

18 Krishnamurthy S, Mathews K, McClure S, et al. Multiinstitutional comparison of whole slide digital imaging and optical microscopy for interpretation of hematoxylin-eosin-stained breast tissue sections. Arch Pathol Lab Med 2013;137:1733-1739.

19 Hale CS, Qian M, Ma MW, et al. Mitotic rate in melanoma: prognostic value of immunostaining and computer-assisted image analysis. Am J Surg Pathol 2013;37:882-889.

20 Wich LG, Hamilton HK, Shapiro RL, et al. Developing a multidisciplinary prospective melanoma biospecimen repository to advance translational research. Am J Transl Res 2009;1:35-43.

21 Leiter U, Marghoob AA, Lasithiotakis K, et al. Costs of the detection of metastases and follow-up examinations in cutaneous melanoma. Melanoma Res 2009;19:50-57.

22 Abbasi NR, Yancovitz M, Gutkowicz-Krusin D, et al. Utility of lesion diameter in the clinical diagnosis of cutaneous melanoma. Arch Dermatol 2008;144:469-474.

23 Wang M, Zhang Z, Zhu J, et al. Tumour diameter is a predictor of mesorectal and mesenteric lymph node metastases in anorectal melanoma. Colorectal Dis 2013;15:1086-1092.

24 Maley A, Rhodes AR. Cutaneous melanoma: preoperative tumor diameter in a general dermatology outpatient setting. Dermatol Surg 2014;40:446-454.

25 Kopf AW, Rodriguez-Sains RS, Rigel DS, et al. 'Small' melanomas: relation of prognostic variables to diameter of primary superficial spreading melanomas. J Dermatol Surg Oncol 1982;8:765-770.

26 Veronesi U, Adamus J, Bandiera DC, et al. Stage I melanoma of the limbs. Immediate versus delayed node dissection. Tumori 1980;66:373-396.

27 Davis NC, McLeod GR, Beardmore GL, et al. Primary cutaneous melanoma: a report from the Queensland melanoma project. CA Cancer J Clin 1976;26:80-107.

28 Cady B. Changinc concepts in malignant melanoma. Med Clin North Am 1975;59:301-308.

29 Mackie RM, Carfrae DC, Cochran AJ. Assessment of prognosis in patients with malignant melanoma. Lancet 1972;2:455-456.

30 Breslow A. Thickness, cross-sectional areas and depth of invasion in the prognosis of cutaneous melanoma. Ann Surg 1970;172:902-908.

31 Dauendorffer JN, Bastuji-Garin S, Guero S, et al. Shrinkage of skin excision specimens: formalin fixation is not the culprit. Br J Dermatol 2009;160: 810-814.

32 Blasco-Morente G, Garrido-Colmenero C, Perez-Lopez I, et al. Study of shrinkage of cutaneous surgical specimens. J Cutan Pathol 2015;42:253-257.

33 Barnhill RL, Katzen J, Spatz A, et al. The importance of mitotic rate as a prognostic factor for localized cutaneous melanoma. J Cutan Pathol 2005;32: 268-273.

34 Azzola MF, Shaw HM, Thompson JF, et al. Tumor mitotic rate is a more powerful prognostic indicator than ulceration in patients with primary cutaneous melanoma: an analysis of 3661 patients from a single center. Cancer 2003;97:1488-1498.

35 Francken AB, Shaw HM, Thompson JF, et al. The prognostic importance of tumor mitotic rate confirmed in 1317 patients with primary cutaneous melanoma and long follow-up. Ann Surg Oncol 2004;11:426-433.

36 Thompson JF, Soong SJ, Balch CM, et al. Prognostic significance of mitotic rate in localized primary cutaneous melanoma: an analysis of patients in the multi-institutional American Joint Committee on Cancer melanoma staging database. J Clin Oncol 2011;29: 2199-2205. 